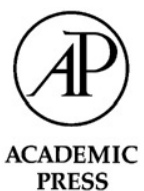

\title{
Does phase cycling work for nuclei experiencing strong quadrupolar couplings?
}

\author{
Philip J. Grandinetti* \\ Department of Chemistry, The Ohio State University, 120 W. 18th Avenue, Columbus, OH 43210-1173, \\ USA
}

Received May 30, 2002; revised September 13, 2002

\begin{abstract}
The question of whether the phase cycling can still be used to select coherence transfer pathways in spin systems experiencing a tilting of its eigenstates away from the Zeeman eigenstates due to strong couplings was investigated theoretically. Based on the analysis presented it is concluded that conventional phase cycling is still a valid approach for selecting a coherence transfer pathway signal, although changes in pathway efficiencies can occur as the mechanisms for excitation and detection of coherences are affected by the tilting of the eigenstates.
\end{abstract}

(C) 2003 Elsevier Science (USA). All rights reserved.

\section{Introduction}

It is generally assumed that phase cycling can be used to select specific coherence pathways during a multiple pulse sequence, even if the spin system is experiencing couplings strong enough that the nuclear spin eigenstates are tilted away from the Zeeman eigenstates. In practice, this seems to be a working approximation. In theory, however, the consequences of this tilting on the coherence transfer pathway selection process are not completely clear. Will it allow signals from other pathways to "leak" into an otherwise, properly phase cycled experiment? How will coherence transfer pathway efficiencies be affected? What changes, if any, are needed in designing phase cycles? Because of the increasing popularity in exploiting NMR of quadrupolar nuclei as structural probes with experiments like DAS [1,2], MQ-MAS

\footnotetext{
*Fax: 614-292-1685.

E-mail address: grandinetti.1@osu.edu.
} 
[3-5], ST-MAS [6], and related sequences these issues should be clarified. In the text below, I review the relevant aspects of higher order effects in NMR, along with certain aspects of phase cycling for coherence transfer pathway selection. Once a unified picture is developed I then explain why the answer to the title question is "yes", and discuss some caveats that may need to be considered when working in such cases.

\section{Theoretical analysis}

\subsection{Defining the frame}

The most natural frame in which to describe an experiment in spectroscopy is the diagonal frame of the unperturbed Hamiltonian, which in magnetic resonance arises from the couplings between the nuclear moments (both magnetic and electric) and surrounding static or time-averaged fields. Another natural choice would be the orientation of the laboratory measuring device, which in magnetic resonance is orientation of the rf coil.

I will define the laboratory frame by orienting the direction of the $x$-axis along the long axis of the rf coil; writing the rf Hamiltonian in that frame as

$$
\mathbf{H}_{r f}\left(\phi_{r f}, t\right)=2 \omega_{1} \cos \left(\omega_{r f} t+\phi_{r f}\right) \mathbf{I}_{X} .
$$

I make this definition using $x$ instead of $z$ because in high-field NMR that is where the rf coil typically excites and detects coherences in the laboratory frame. In the absence of rf irradiation the spins evolve under an unperturbed Hamiltonian, whose representation in its diagonal frame, $\mathbf{D}_{S}$, is related to our laboratory frame representation, $\mathbf{H}_{S}$, by

$$
\mathbf{H}_{S}=\mathbf{V D}_{S} \mathbf{V}^{\dagger}
$$

where $\mathbf{V}$ is a unitary transformation between the two frames. In the weak coupling, high-field limit of NMR these two frames coincide.

If the Zeeman interaction is the dominant interaction in $\mathbf{H}_{S}$ then we find that our treatment can be further simplified by moving into a frame rotating about the $z$-axis of the diagonal frame. We call this the "rotating tilted" frame [7], and all operators defined in this frame will carry a circle superscript. The density operator in the rotating tilted frame, $\rho^{\circ}(t)$, is thus related to the laboratory frame density operator by

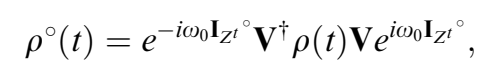

which evolves under an effective Hamiltonian given by

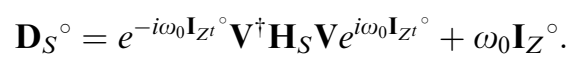

In the discussion that follows I assume that the Zeeman interaction is the dominant interaction in $\mathbf{H}_{S}$, and there are no degeneracies in $\mathbf{H}_{S}$. 


\subsection{The equilibrium density operator}

In the high-temperature approximation, we take the equilibrium density operator to be proportional to the unperturbed Hamiltonian. Generally, one expects an equilibrium density operator to be diagonal (i.e., no coherences present). This will certainly be the case when the equilibrium density operator is written in the diagonal frame of the unperturbed Hamiltonian. When expressed in the laboratory frame, however, the equilibrium density operator may contain offdiagonal elements that could be mistaken for coherences. To avoid this unnecessary complication, we follow the evolution of coherences and, also label them, in the diagonal frame of the unperturbed Hamiltonian, or, if appropriate, in the rotating tilted frame.

In the weak coupling, high-field limit of NMR we have an equilibrium density operator that, to a good approximation, is given by $\rho_{e q}{ }^{\circ}=\mathbf{I}_{Z}{ }^{\circ}$. In the presence of strong couplings, we find that the equilibrium density operator in the rotating tilted frame can decompose into a linear combination of irreducible tensor operators $[8,9]$ of many possible ranks, $l$, but all of order $p=0$, that is,

$$
\rho_{e q}{ }^{\circ}=\sum_{l} a_{l} \mathbf{T}_{l, 0}{ }^{\circ} .
$$

Here again, I use the circle superscript to emphasize that the tensor operators are defined with respect to the rotating tilted frame. In general, we cannot expect spin systems that are identical, except for their orientation within the same sample, to have identical equilibrium density operators in the presence of strong couplings. In other words, the rotating tilted frame for different spin systems in the same sample may have different orientations with respect to the laboratory frame, particularly in a polycrystalline sample. Additionally, the coefficients, $a_{l}$, may depend on the relative orientation of the tilted frame with respect to the laboratory frame. In the strong coupling, zero-field limit of NQR, however, the coefficients, $a_{l}$, will become independent of the laboratory frame orientation.

\subsection{Detection}

The signal detected by the receiver coil (also along $x$ in the laboratory frame) is given by

$$
S(t)=\operatorname{Tr}\left\{\rho(t) \mathbf{I}_{X}\right\} e^{i \phi_{R}}=\operatorname{Tr}\left\{\rho^{\circ}(t) \mathbf{I}_{X}^{\circ}(t)\right\} e^{i \phi_{R}},
$$

where $\phi_{R}$ is the receiver phase, and

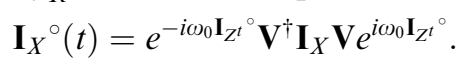

Within the NMR spectrometer only signal frequencies oscillating within a given bandwidth at the detector frequency are observed. This process can be included in our theoretical description by shifting the frequencies in our calculated signal by $\omega_{R}$ according to

$$
S^{\prime}(t)=\operatorname{Tr}\left\{\rho^{\circ}(t) \mathbf{I}_{X}{ }^{\circ}(t)\right\} e^{i \omega_{R} t} e^{i \phi_{R}},
$$


and subsequently dropping all terms containing oscillations outside the bandwidth of the spectrometer. In the presence of strong couplings, one can generically write the expansion for our laboratory $\mathbf{I}_{X}$ in the rotating tilted frame as

$$
\mathbf{I}_{X}{ }^{\circ}(t)=\sum_{l, m} a_{l, m} \mathbf{T}_{l, m}^{\circ} e^{-i m \omega_{0} t}
$$

Setting the receiver frequency, $\omega_{R}$, near the single quantum transition frequency, substituting Eq. (3) into Eq. (2), and, removing oscillations outside the bandwidth of the spectrometer, one obtains

$$
S^{\prime}(t)=\sum_{l} a_{l, 1} \operatorname{Tr}\left\{\rho^{\circ}(t) \mathbf{T}_{l, 1}^{\circ} e^{i \Delta \omega_{R} t}\right\} e^{-i \phi_{R}}
$$

where $\Delta \omega_{R}=\omega_{R}-\omega_{0}$. In the weak coupling, high field limit only first-rank single quantum coherences (i.e., $\mathbf{I}_{-}$) will be detected. In the presence of strong couplings we still find only single quantum coherences detected by the receiver coil, but now they can be of higher rank.

\subsection{Excitation}

During rf excitation the spins will evolve under the combined Hamiltonian

$$
\mathbf{H}\left(\phi_{r f}, t\right)=\mathbf{H}_{S}+\mathbf{H}_{r f}\left(\phi_{r f}, t\right) .
$$

If one assumes that the magnitude of the rf term is small compared to the unperturbed Hamiltonian, i.e., $\left\|\mathbf{H}_{S}\right\| \gg\left\|\mathbf{H}_{r f}(t)\right\|$ at all times, then the static diagonalization of $\mathbf{H}_{S}$ can be used to transform the time-dependent rf Hamiltonian into the rotating tilted frame, yielding

$$
\mathbf{H}_{r f}{ }^{\circ}\left(\phi_{r f}, t\right)=2 \omega_{1} \cos \left(\omega_{r f} t+\phi_{r f}\right) \mathbf{I}_{X}{ }^{\circ}(t) .
$$

In the presence of strong couplings, an rf pulse is capable of generating a greater variety of coherence transfers. For example, the direct excitation of multiple quantum (or overtone) coherences is possible in the presence of strong couplings by setting the irradiation frequency, $\omega_{r f}$, near the overtone transition frequency [10].

Setting $\omega_{r f}$ near the single quantum (fundamental) transitions, substituting Eq. (3) into Eq. (6), and eliminating the fast oscillating terms one obtains

$$
\mathbf{H}_{r f}{ }^{\circ}\left(\phi_{r f}, t\right)=\omega_{1} \sum_{l}\left\{a_{l,-1} \mathbf{T}_{l,-1}{ }^{\circ} e^{-i \Delta \omega_{r f} t} e^{i \phi_{r f}}+a_{l, 1} \mathbf{T}_{l, 1}{ }^{\circ} e^{i \Delta \omega_{r f} t} e^{-i \phi_{r f}}\right\},
$$

where $\Delta \omega_{r f}=\omega_{r f}-\omega_{0}$. This term, by itself, would produce single quantum excitation. In the weak coupling, high-field limit this Hamiltonian would contain only first-rank single quantum operators. In the presence of strong couplings it may contain higher rank single quantum operators, which could provide additional opportunities for manipulating coherences during the rf pulse. 


\subsection{Coherence transfer pathway selection with phase cycling}

The density operator can be expanded in terms of the irreducible tensor operators of the group $\mathrm{SO}(3)$ with rank $l$ and order $p$,

$$
\rho^{\circ}(t)=\sum_{l, p} a_{l, p}(t) \mathbf{T}_{l, p}^{\circ} .
$$

Coherences described by irreducible tensor operators of the same rank transform amongst themselves under rotations about $x, y$, or $z$. This is a key feature that allows us to ignore the coherence rank in the coherence transfer pathway approach and group all coherences of the same order together into a generic coherence, ${ }^{1} \mathbf{T}_{p}{ }^{\circ}$, and write our density operator expansion as

$$
\rho^{\circ}(t)=\sum_{p} b_{p}(t) \mathbf{T}_{p}^{\circ} .
$$

In the absence of rf irradiation, there will be no change in coherence order, $p$, as this density operator evolves; only the coefficients $b_{p}(t)$ will be affected by unperturbed evolution.

To illustrate the concept of a coherence transfer pathway [11-13] let us consider a single spin $1 / 2$ system and consider the generic two-pulse NMR experiment. If one wrote out the full density operator for the entire two pulse experiment for arbitrary pulse phases and lengths, the resulting expansion would quickly become complicated. Starting with $\rho(0)=\mathbf{I}_{Z}$, as shown in Fig. 1, terms in the density operator expansion grow geometrically with each pulse. After two pulses there will be nine different terms in the density operator expansion, each multiplied by coefficients that carry the history of each term. Tracing the path of each of these terms back to the initial $\rho(0)$ term reveals all the possible transition frequency modulations that can be present in a signal. These paths represent different NMR experiments, and are graphically represented as different coherence transfer pathways.

With phase cycling, signals from different coherence transfer pathways can be separated by taking advantage of a Hamiltonian during the rf pulse that obeys the relationships:

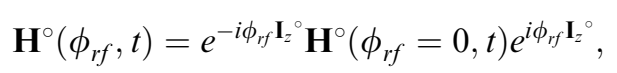

from which it follows that

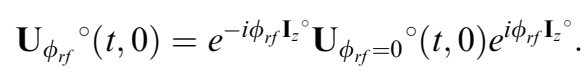

This is demonstrated by using Eq. (9) to derive the generic expression

$$
\mathbf{U}_{\phi_{1}}(t) \mathbf{T}_{p_{0}}{ }^{\circ} \mathbf{U}_{\phi_{1}}^{\dagger}(t)=\sum_{p_{1}} c_{p_{0}, p_{1}}(t) \mathbf{T}_{p_{1}}{ }^{\circ} e^{-i \Delta p_{1} \phi_{1}},
$$

\footnotetext{
${ }^{1}$ These correspond to the irreducible representations of the group $C_{\infty}$.
} 

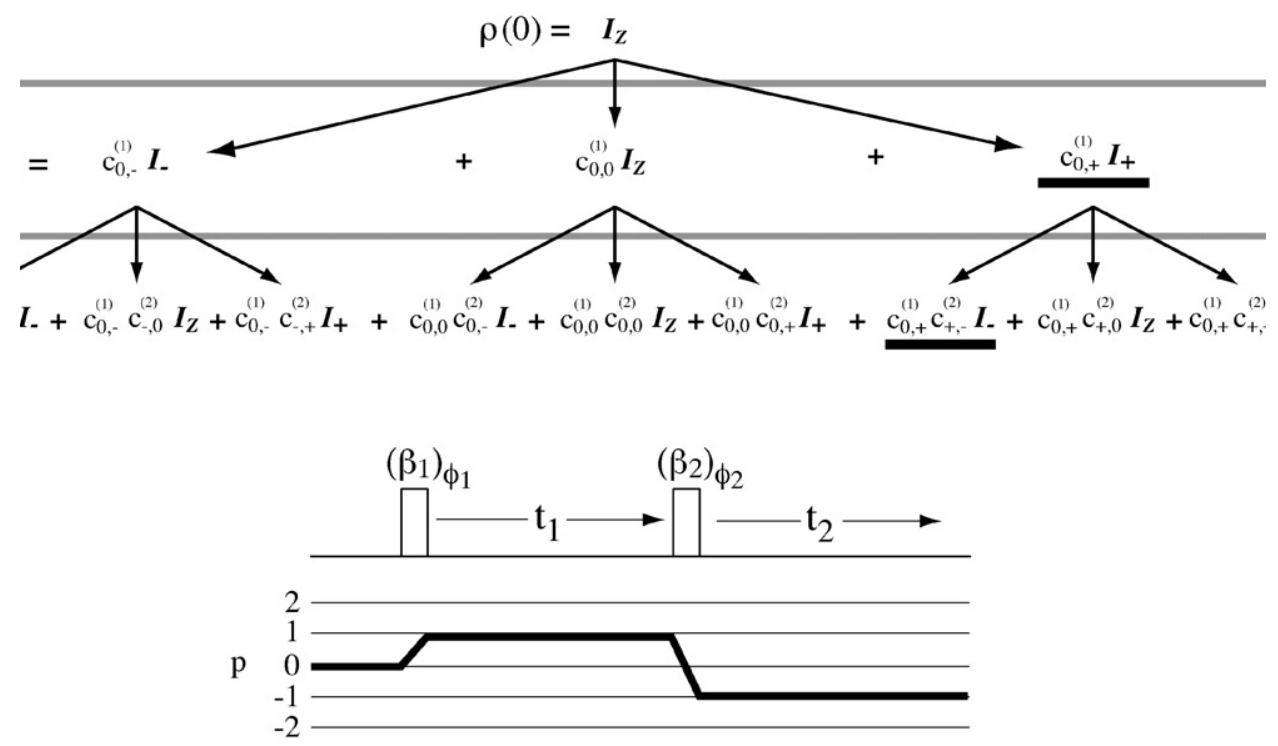

(b)

Fig. 1. (A) Illustration of the propagation of the density operator of a single spin $1 / 2$ system in a two pulse experiment. (B) Coherence transfer pathway associated with the underlined density operator terms in (A).

for the evolution of $\mathbf{T}_{p_{0}}{ }^{\circ}$ under a pulse with $\mathrm{rf}$ phase $\phi_{1}$. Here $\Delta p_{1}=p_{1}-p_{0}$. By performing a Fourier transform with respect to the rf pulse phase, $\phi_{1}$, the signals associated with different coherence transfer paths $\Delta p_{1}$ can be separated [14,15]. Alternatively, the signals from undesired pathways can be averaged to zero during signal summation by phase shifting the rf pulse phases and then phase shifting the receiver phase according to

$$
\phi_{R}=-\sum_{n=1}^{N_{T}} \Delta p_{n}^{(\text {desired })} \phi_{n},
$$

before addition into the signal sum $[12,13]$. Here $N_{T}$ is the number of coherence transfer steps during a pulse sequence, $\Delta p_{n}^{\text {(desired) }}$ is the desired coherence transfer during step $n$, and $\phi_{n}$ is the phase of the $\mathrm{rf}$ excitation during step $n$. With the proper cycling of $\phi_{n}$ and $\phi_{R}$ only the signal from the desired pathway survives the averaging process.

I now address the primary objective of this work by first noting that Eq. (7) satisfies the relationship in Eq. (8). Thus, despite the eigenstate tilting, a Fourier transform of the rf pulse phase will still provide a separation of signals from pathways experiencing different changes in coherence order. Two additional assumptions in the coherence transfer pathway approach is that every pathway begins at $p=0$ and ends at $p=-1$. These assumptions also remain true when strong 
couplings are present. Assumptions, however, that the pathway's initial $p=0$ coherence and final $p=-1$ coherence are first rank are not necessarily true in the presence of strong couplings. A separation of the different rank contributions to a pathway would be possible if one could apply a systematic second rotation about the $x$ - or $y$-axis of the rotating tilted frame, in the same manner that phase cycling effects a systematic rotation about the $z$-axis. This approach has been demonstrated for coupled spin 1/2 nuclei by Suter and Pearson [16]. Applying such an approach in the presence of the strong couplings of quadrupolar nuclei may be problematic, particularly, since a pure rotation about the $x$ - or $y$-axis would require rf field strengths in excess of the strong couplings experienced by the spin system.

\subsection{NMR of quadrupolar nuclei experiencing second-order broadenings}

In this section I consider the specific NMR example of a quadrupolar nucleus with a first-order tilting of its eigenstates away from the Zeeman eigenstates. I adopt the static perturbation theory (SPT) approach where the unperturbed Hamiltonian is divided into two parts:

$$
\mathbf{H}_{S}=\mathbf{H}^{(0)}+\mathbf{H}^{(1)} .
$$

Here $\mathbf{H}^{(0)}$ is associated with the Zeeman interaction

$$
\mathbf{H}^{(0)}=\mathbf{H}_{Z}=-\omega_{0} \mathbf{I}_{Z},
$$

and $\mathbf{H}^{(1)}$ with the quadrupolar interaction

$$
\mathbf{H}^{(1)}=\mathbf{H}_{Q}=\sum_{m} \mathbf{H}_{m}
$$

where $\mathbf{H}_{m}=\omega_{Q}(-1)^{m} A_{2-m}\left(\Omega_{q}\right) \mathbf{T}_{2, m}$. Using the SPT approach as outlined by Goldman et al. [7], both $\mathbf{V}$ and $\mathbf{D}_{S}$ can written in terms of a series expansion,

$$
\mathbf{V}=\mathbf{1}+\mathbf{V}^{(1)}+\mathbf{V}^{(2)}+\cdots \quad \text { and } \quad \mathbf{D}_{S}=\mathbf{H}^{(0)}+\mathbf{D}^{(1)}+\mathbf{D}^{(2)}+\cdots,
$$

with each correction expanded in terms of irreducible tensor operators. To secondorder one has

$$
\begin{aligned}
& \mathbf{V}^{(1)}=-\frac{\omega_{q}}{\omega_{0}} \sum_{m \neq 0}(-1)^{m} \frac{A_{2,-m}\left(\Omega_{q}\right) \mathbf{T}_{2, m}}{m}, \\
& \mathbf{D}^{(1)}=\omega_{q} A_{2,0}\left(\Omega_{q}\right) \mathbf{T}_{2,0} \circ
\end{aligned}
$$


and

$$
\mathbf{D}^{(2)}=-\frac{\omega_{q}^{2}}{\omega_{0}} \sum_{L=0,2,4} \sum_{J=1,3} a_{L, J} \mathscr{A}_{L, 0}\left(\Omega_{q}\right) \mathscr{T}_{J, 0}^{\circ}
$$

where the coefficients and operators used in Eq. (14) are defined in the appendix.

Based on the equations above one can generically expand the equilibrium density operator as

$$
\rho_{e q}{ }^{\circ} \approx \sum_{l=0}^{3} a_{l} \mathbf{T}_{l, 0}{ }^{\circ} .
$$

Although this expression contains terms up to third rank, the first-rank contribution, or what is more commonly called Zeeman order, will dominate this expansion. The second-rank first-order quadrupolar Hamiltonian, or what might be termed quadrupolar order, will be the next largest contribution, with a fractional contribution of approximately $\omega_{q} /\left(\omega_{0}+\omega_{q}\right)$. Although I have not considered sample motion in the discussion, with magic-angle spinning the contribution from the first-order quadrupolar to the equilibrium density operator may be diminished, if not averaged away completely. Contributions from the second-order quadrupolar Hamiltonian will contribute substantially less, and likely have a negligible contribution, although they will not average to zero under MAS.

Solving for $\mathbf{I}_{X}^{\circ}(t)$ one obtains

$$
\begin{aligned}
\mathbf{I}_{X}{ }^{\circ}= & \frac{1}{2}\left(\mathbf{I}_{+}{ }^{\circ} e^{-i \omega_{0} t}+\mathbf{I}_{-}{ }^{\circ} e^{i \omega_{0} t}\right) \\
& +\frac{\omega_{Q}}{2 \omega_{0}} \sum_{m \neq 0} \frac{(-1)^{m} A_{2,-m} e^{-i m \omega_{0} t}}{m}\left[\sqrt{6-m^{2}-m} \mathbf{T}_{2, m+1}{ }^{\circ} e^{-i \omega_{0} t}\right. \\
& \left.+\sqrt{6-m^{2}+m} \mathbf{T}_{2, m-1}{ }^{i \omega_{0} t}\right] .
\end{aligned}
$$

Using Eq. (15) in Eq. (6), setting $\omega_{r f}$ near the single quantum transition frequency, the rf Hamiltonian in the rotating tilted frame after eliminating the fast oscillating terms becomes

$$
\begin{aligned}
\mathbf{H}_{r f}{ }^{\circ}\left(\phi_{r f}, t\right)= & \frac{\omega_{1}}{2}\left[\mathbf{I}_{-}{ }^{\circ} e^{-i \Delta \omega_{r f} t} e^{-i \phi_{r f}}+\mathbf{I}_{+}{ }^{\circ} e^{i \Delta \omega_{r f} t} e^{i \phi_{r f}}\right] \\
& -\frac{\omega_{1} \omega_{Q}}{2 \omega_{0}}\left[A_{2,2}\left(\Omega_{q}\right) \mathbf{T}_{2,-1} e^{i \Delta \omega_{r f} t} e^{-i \phi_{r f}}\right. \\
& -A_{2,-2}\left(\Omega_{q}\right) \mathbf{T}_{2,1}{ }^{\circ} e^{-i \Delta \omega_{r f} t} e^{\left.i \phi_{r f}\right] .}
\end{aligned}
$$

As expected this expression satisfies the relationship in Eq. (8). The magnitude of the second-rank terms is approximately $\omega_{1} \omega_{q} / \omega_{0}$. In the direct excitation of coherences, the second-rank term is usually negligible.

Similarly, substituting Eq. (15) into Eq. (2), setting $\omega_{R}$ near the single quantum transition frequency, and eliminating the signal oscillations outside the bandwidth of 
the spectrometer, one obtains

$$
S^{\prime}(t, 0)=\operatorname{Tr}\left\{\rho^{\circ}(t) \mathbf{I}_{+}^{\circ} e^{i \Delta \omega_{R} t}\right\} e^{i \phi_{R}}+\frac{\omega_{Q} A_{2,-2}\left(\Omega_{q}\right)}{2 \omega_{0}} \operatorname{Tr}\left\{\rho^{\circ}(t) \mathbf{T}_{2,1}{ }^{\circ} e^{i \Delta \omega_{R} t}\right\} e^{i \phi_{R}} .
$$

While it is still true that only $p=-1$ coherences are detected, the effect of the tilting is to add into the signal a small contribution from a second observable of $\mathbf{T}_{2,1}{ }^{\circ}$. The size of the contribution, is on the order of $\omega_{Q} / \omega_{0}$, and at best, might be on the order a few percent.

\subsection{Overtone NMR of quadrupolar nuclei}

In the previous sections I assumed the transmitter and receiver would be operating in the vicinity of the single quantum (fundamental) transitions (i.e., the $\Delta m= \pm 1$ transitions as defined by the eigenstates of the Zeeman Hamiltonian). In this section I consider placing the transmitter and receiver in the vicinity of the overtone transition $[10,17,18]$, which leads to the creation of double quantum coherences in the rotating tilted frame. As stated earlier, coherences are labeled as seen in the rotating tilted frame, regardless of how they are excited.

Using Eq. (15) in Eq. (6), setting $\omega_{r f}$ near the overtone transition frequency, the rf Hamiltonian in the rotating tilted frame after eliminating the fast oscillating terms becomes

$$
\begin{aligned}
\mathbf{H}_{r f}{ }^{\circ}\left(\phi_{r f}, t\right)= & \frac{\omega_{1} \omega_{Q}}{\omega_{0}}\left[A_{2,-1}\left(\Omega_{q}\right) \mathbf{T}_{2,2}{ }^{\circ} e^{i \Delta \omega_{r f} t} e^{i \phi_{r f}}\right. \\
& \left.-A_{2,1}\left(\Omega_{q}\right) \mathbf{T}_{2,-2} e^{-i \Delta \omega_{r f} t} e^{-i \phi_{r f}}\right] .
\end{aligned}
$$

As expected, this rf Hamiltonian, by itself, can directly excite double quantum coherence. Similarly, substituting Eq. (15) into Eq. (2), setting $\omega_{R}$ near the overtone transition frequency, and eliminating the signal oscillations outside the bandwidth of the spectrometer, one obtains

$$
S^{\prime}(t, 0)=\frac{\omega_{Q} A_{2-1}\left(\Omega_{q}\right)}{2 \omega_{0}} \operatorname{Tr}\left\{\rho^{\circ}(t) \mathbf{T}_{2,2} e^{i \Delta \omega_{R} t}\right\} e^{-i \phi_{R}} .
$$

Here one finds, again as expected, that double quantum coherences can be directly detected.

At first glance, one might conclude from Eqs. (18) and (19) that phase cycling will not perform as expected in overtone NMR. For example, Eq. (18) does not satisfy the relationship in Eqs. (8) and (19) requires that only $p=-2$ coherence are detected in our coherence transfer pathways. In practice, these differences have little or no effect. Instead of Eq. (8) one finds that the Hamiltonian during the rf pulse follows:

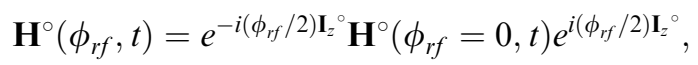

from which it follows that

$$
\mathbf{U}_{\phi_{r f}}{ }^{\circ}(t, 0)=e^{-i\left(\phi_{r f} / 2\right) \mathbf{I}_{z}{ }^{\circ}} \mathbf{U}_{\phi_{r f}=0}{ }^{\circ}(t, 0) e^{i\left(\phi_{r f} / 2\right) \mathbf{I}_{z}{ }^{\circ}}
$$




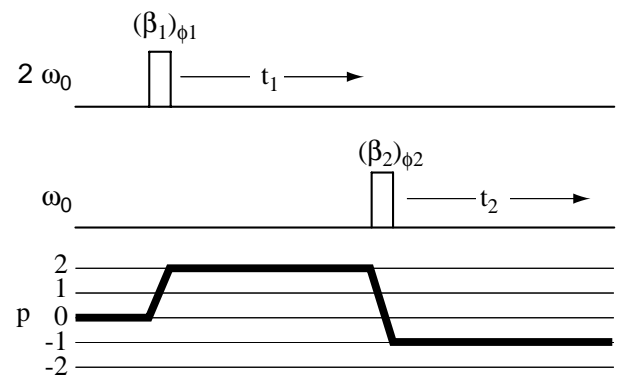

Fig. 2. Double resonance overtone/fundamental correlation experiment.

and

$$
\mathbf{U}_{\phi_{1}}(t) \mathbf{T}_{p_{0}}^{\circ} \mathbf{U}_{\phi_{1}}^{\dagger}(t)=\sum_{p_{1}} c_{p_{0}, p_{1}}(t) \mathbf{T}_{p_{1}}^{\circ} e^{-i\left(\Delta p_{1} / 2\right) \phi_{1}} .
$$

Thus, in overtone NMR a Fourier transform with respect to the pulse rf phase, $\phi_{1}$ will still separate signals from different coherence transfer paths $\Delta p_{1}$, but now the observed change in coherence order will appear at half its actual value. Therefore, signals from undesired pathways can still be averaged to zero during signal summation by cycling the rf pulse phases and the receiver phase according to

$$
\phi_{R}=-\frac{1}{2} \sum_{n} \Delta p_{n}^{(\text {desired })} \phi_{n}
$$

One might argue that the factor of $1 / 2$ in Eq. (23) is simply a consequence of labeling coherences created and observed during the overtone experiment as double quantum instead of single quantum (i.e., from one photon excitation), and with a properly chosen frame an expression like Eq. (11) could be obtained. In a double resonance experiment involving both overtone and fundamental transitions, however, a difference between Eqs. (11) and (23) would still need to be taken into account. Take, as an example, the double resonance experiment, shown in Fig. 2, correlating overtone and fundamental transition frequencies. For this experiment one would need to use Eq. (23) when applying rf pulses at $2 \omega_{0}$, and Eq. (11) for rf pulses at $\omega_{0}$. In this case, the relationship between receiver phase and pulse phases needed to select the pathway shown in Fig. 2 would be

$$
\phi_{R}=-\frac{1}{2} \Delta p_{1}^{\text {(desired) }} \phi_{1}-\Delta p_{2}^{(\text {desired })} \phi_{2}=-\phi_{1}+3 \phi_{2} .
$$

On the other hand, if one desired the same coherence transfer pathway in a two-pulse sequence with both pulses are applied at $\omega_{0}$, then only Eq. (11) would be applied. In this case the phase cycle relationship $\phi_{R}=-2 \phi_{1}+3 \phi_{2}$ would be needed to select the same pathway. 


\section{Conclusions}

I have theoretically investigated the question of whether conventional phase cycling can still be used to select coherence transfer pathways in spin systems with eigenstates tilted away from the Zeeman eigenstates. Based on the analysis presented here I conclude that it is still a valid approach. A key concept is clarifying this situation is that coherences should be defined in the diagonal frame of the unperturbed, time-independent or time-averaged Hamiltonian. In the high-field, weak coupling limit, the diagonal frame coincides with the laboratory frame, and there is no need to transform operators when moving between the two frames. In the strong coupling situation, however, these two frame no longer coincide, and thus all observables and contributions to the Hamiltonian defined in the laboratory frame should be transformed into the rotating tilted frame, where their relationships to the experiment's coherence pathway are more easily defined. The orientation of this rotating tilted frame will be a function of the sample orientation. These are, of course, well-known effects of the tilting of the spin eigenstates away from the Zeeman eigenstates.

With regard to phase cycling in the strong coupling case certain assumptions about (1) the initial density operator, (2) the observable operator, and (3) the excitation capabilities of the rf pulse, need to be modified. In particular, the initial density operator may be decomposed in terms of irreducible tensor operators of rank higher than $\mathbf{I}_{z}^{\circ}$. The observable operator, while remaining a single quantum operator, may similarly contain higher rank observables than $\mathbf{I}_{+}{ }^{\circ}$. Finally, the rf Hamiltonian, like the observable, may contain multiple rank single quantum operators. The important conclusion from this analysis is that all the essential concepts associated with phase cycling to select coherence orders, and coherence transfer pathways still apply, and can be used with little or no modification. For nuclei experiencing second-order quadrupolar broadenings the effect of the tilting appears to be negligible for popular sequences like DAS, MQ-MAS, and ST-MAS.

The issue of sample motion was not addressed. With sample motion the orientation of the diagonal frame with respect to the laboratory frame may become time dependent. When there is only a slight tilting of the eigenstates away from the Zeeman eigenstates, however, the effect of this motion on coherence transfer pathway (ending at $p=-1$ ) signals will likely be minimal.

\section{Acknowledgments}

This material is based upon work supported in part by the National Science Foundation under Grant CHE 0111109, and the U.S. Department of Energy. Any opinions, findings and conclusions or recommendations expressed in this material are those of the author(s) and do not necessarily reflect the views of the National Science Foundation (NSF). 


\section{Appendix}

The coefficients and operators used in Eq. (14) are

$$
a_{L, J}=2 \sum_{m>0} \frac{\langle L 0 \mid 22 m-m\rangle\langle J 0 \mid 22 m-m\rangle}{m} .
$$

The tensor $\mathscr{A}_{L, n}\left(\Omega_{q}\right)$ is related to the principal values of the $A_{L, n}\left(\Omega_{q}\right)$ tensor by

$$
\mathscr{A}_{L, n}\left(\Omega_{q}\right)=\sum_{n^{\prime}=-L}^{L} \mathscr{D}_{n^{\prime}, n}^{(L)}\left(\Omega_{q}\right) \sigma_{L, n^{\prime}}
$$

with

$$
\sigma_{L, n}=\sum_{m}\langle L n \mid 22 m n-m\rangle \rho_{2, k} \rho_{2, n-k},
$$

where $\rho_{2,0}=1 / \sqrt{6}$ and $\rho_{2, \pm 2}=\rho_{2,0} \cdot \eta_{q} / \sqrt{6}$. Finally, using the Wigner-Eckert theorem [9], the $\mathscr{T}_{l, k}$ are related to our originally defined irreducible tensor operators, $\mathbf{T}_{l, k}$ according to

$$
\mathscr{T}_{1,0}=\sqrt{\frac{2}{5}}[I(I+1)-3 / 4] \mathbf{T}_{1,0} \quad \text { and } \quad \mathscr{T}_{3,0}=-2 \mathbf{T}_{3,0} .
$$

\section{References}

[1] K.T. Mueller, B.Q. Sun, G.C. Chingas, J.W. Zwanziger, T. Terao, A. Pines, Dynamic-angle spinning of quadrupolar nuclei, J. Magn. Reson. 86 (1990) 470.

[2] P.J. Grandinetti, J.H. Baltisberger, A. Llor, Y.K. Lee, U. Werner, M.A. Eastman, A. Pines, Pure absorption-mode lineshapes and sensitivity in two-dimensional dynamic angle spinning NMR, J. Magn. Reson. A 103 (1993) 72-81.

[3] L. Frydman, J.S. Harwood, Isotropic spectra of half-integer quadrupolar spins from bidimensional magic-angle spinning NMR, J. Am. Chem. Soc. 117 (1995) 5367-5369.

[4] A. Medek, J.S. Harwood, L. Frydman, Multiple-quantum magic-angle spinning NMR: a new method for the study of quadrupolar nuclei in solids, J. Am. Chem. Soc. 117 (1995) 12779-12787.

[5] D. Massiot, B. Touzo, D. Trumeau, J.P. Coutures, J. Virlet, P. Florian, P.J. Grandinetti, Twodimensional magic-angle spinning isotropic reconstruction sequences for quadrupolar nuclei, Solid State NMR 6 (1996) 73-83.

[6] Z. Gan, Isotropic NMR spectra of half-integer quadrupolar nuclei using satellite transitions and magic-angle spinning, J. Am. Chem. Soc. 122 (2000) 3242-3243.

[7] M. Goldman, P.J. Grandinetti, A. Llor, Z. Olejniczak, J.R. Sachleben, J.W. Zwanziger, Theoretical aspects of higher-order truncations in solid-state NMR, J. Chem. Phys. 97 (1992) 8947-8960.

[8] B.L. Silver, Irreducible Tensor Methods, Academic Press Inc, San Diego, CA, 1976.

[9] M. Weissbluth, Atoms and Molecules, Academic Press, San Diego, CA, 1978.

[10] R. Tycko, S.J. Opella, High resolution ${ }^{14} \mathrm{~N}$ overtone spectroscopy: an approach to natural abundance nitrogen NMR of oriented and polycrystalline systems, J. Am. Chem. Soc. 108 (1986) 3531.

[11] R.R. Ernst, G. Bodenhausen, A. Wokaun, Principles of Nuclear Magnetic Resonance in One and Two Dimensions, Oxford, Oxford, 1987.

[12] G. Bodenhausen, H. Kogler, R.R. Ernst, Selection of coherence-transfer pathways in NMR pulse experiments, J. Magn. Reson. 58 (1984) 370-388.

[13] A.D. Bain, Coherence levels and coherence pathways in NMR. a simple way to design phase cycling procedures, J. Magn. Reson. 56 (1984) 418-427. 
[14] G. Drobny, A. Pines, S. Sinton, D. Weitekamp, D. Wemmer, Fourier transform multiple quantum nuclear magnetic resonance, Symp. Faraday Soc. 13 (1980) 93.

[15] A. Wokaun, R.R. Ernst, Selective detection of multiple quantum transitions in NMR by twodimensional spectroscopy, Chem. Phys. Lett. 52 (1977) 407-412.

[16] D. Suter, J.G. Pearson, Experimental classification of multi-spin coherence under the full rotation group, Chem. Phys. Lett. 144 (4) (1988) 328-332.

[17] R. Tycko, S.J. Opella, Overtone NMR spectroscopy, J. Chem. Phys. 86 (1987) 1761.

[18] R. Tycko, P.L. Stewart, S.J. Opella, Peptide plane orientations determined by fundamental and overtone ${ }^{14}$ N NMR, J. Am. Chem. Soc. 108 (1986) 5419. 